\title{
Les écrits historiques britanniques et la Première Guerre mondiale
}

Writings by British historians on the First World War

John Mullen

\section{(2) OpenEdition}

\section{Journals}

Édition électronique

URL : http://journals.openedition.org/rfcb/232

DOI : $10.4000 /$ rfcb.232

ISSN : 2429-4373

Éditeur

CRECIB - Centre de recherche et d'études en civilisation britannique

\section{Édition imprimée}

Date de publication : 15 janvier 2015

ISSN : 0248-9015

\section{Référence électronique}

John Mullen, "Les écrits historiques britanniques et la Première Guerre mondiale », Revue Française de Civilisation Britannique [En ligne], XX-1 | 2015, mis en ligne le 15 février 2015, consulté le 19 avril 2019. URL : http://journals.openedition.org/rfcb/232 ; DOI : 10.4000/rfcb.232

Ce document a été généré automatiquement le 19 avril 2019

\section{(c) $($ ) $\odot$ EY}

Revue française de civilisation britannique est mis à disposition selon les termes de la licence Creative Commons Attribution - Pas d'Utilisation Commerciale - Pas de Modification 4.0 International. 


\title{
Les écrits historiques britanniques et la Première Guerre mondiale
}

\author{
Writings by British historians on the First World War
}

John Mullen

1 La Première Guerre tient une place unique dans les études historiques. La production de livres est très abondante : chaque mois on publie une trentaine de nouveaux ouvrages en anglais (et une dizaine en français)! Une consultation du site web Amazon.co.uk ${ }^{1}$ révèle un total de 10061 livres en vente dont le descriptif contient l'expression «World War I ». Ce nombre comprend 566 livres pour enfants, 87 livres humoristiques et 1884 livres $\mathrm{d}^{\prime}$ histoire militaire. Les trois meilleures ventes sont les nouveaux livres de Max Hastings ${ }^{2}$ et de Christopher Clarke, ${ }^{3}$ ainsi que les mémoires classiques d'Ernst Junger de $1920 .{ }^{4}$

2 La production d'écrits sur ce conflit est multiforme. La production universitaire reste importante: à titre d'exemple, 103 thèses concernant la Première Guerre ont été soutenues au Royaume Uni depuis l'an 2000. Mais les universités sont loin d'avoir le monopole: le thème fait vendre suffisamment pour permettre à quelques auteurs de gagner leur vie en étudiant en travaillant à leur compte la Grande Guerre ; bien d'autres en font leur violon d'Ingres et une multitude d'associations d'histoire locale ou familiale publie également de nombreux ouvrages. On ne peut tracer une ligne précise entre production savante et populaire. Si les innombrables livres populaires annoncés comme «the unknown story of» ont parfois des défauts - la sélection des sources n'est pas explicitée et la théorisation de leur statut peut manquer - ils contiennent souvent des traitements de sources primaires tout à fait intéressants.

3 Le lectorat des ouvrages est également très varié. Une envie de mieux comprendre l'histoire familiale ou d'approcher de près le vécu de nos ancêtres motive de nombreux lecteurs; d'autres sont des aficionados de la chose militaire. Pour ces deux groupes l'authenticité du récit historique et son individualité sont des valeurs clé. Une bibliothèque municipale choisie au hasard (celle de la ville d'Accrington, population 35 000), contenait dans la section « histoire » environ 500 livres. Un quart de ces livres 
concernait la Première Guerre mondiale, et, au sein de cette sélection, la vaste majorité traitait de la vie quotidienne des soldats ordinaires.

4 Le présent article vise à analyser les écrits historiques sur la Première Guerre en se concentrant sur les publications des 50 dernières années. Ce travail ne prétend évidemment pas à une quelconque exhaustivité, mais constitue une présentation des tendances majeures. Après avoir posé la question de comment catégoriser une bibliographie si abondante, nous regarderons l'effet sur les écrits historiques des grands tournants historiographiques du XXe siècle, notamment ceux de "l'histoire sociale » et de «l'histoire culturelle». Ensuite nous traiterons de quelques débats contemporains avant de finir par une analyse thématique des cent thèses doctorales sur la Grande Guerre soutenues au Royaume Uni depuis l'an 2000.

\section{Questionnements}

5 Dans son ouvrage Qu'est-ce que l'histoire ${ }^{5}$, un recueil de conférences édité pour la première fois en 1961 et jouissant d'une influence très large, E. H. Carr soutient que la tâche centrale de l'historien est de chercher à savoir "pourquoi ». "Faire de l'histoire, c'est rechercher des causes » écrit-il ${ }^{6}$. Mais le choix des causes à rechercher n'est pas un choix neutre. L'historien peut s'interroger sur les causes économiques de la Première Guerre, sur la motivation des soldats volontaires, sur ce qui a rendu possible de telles violences chez des êtres précédemment non-violents ou encore sur les raisons du ralliement du féminisme et du syndicalisme à la guerre. D'autres pourraient s'intéresser aux causes des changements dans la tactique des généraux, aux causes d'une victoire ou d'une défaite dans une bataille particulière, à l'utilisation des chars ou du gaz toxique. Il n'y a pas consensus concernant l'importance des différentes questions. Le choix des questions définit l'historien.

6 Certains ouvrages tentent encore de traiter l'ensemble de l'histoire militaire de la guerre, tels que l'ouvrage de Gary Sheffield, Forgotten Victory, ${ }^{7}$ ou bien de l'histoire de la vie en Grande-Bretagne durant la guerre comme le récent livre d'Adrian Gregory ${ }^{8}$ que les critiques ont comparé à l'influent ouvrage d'Arthur Marwick'. Cependant, un terrain autant labouré encourage le traitement de questions très précises, et l'histoire par objet est courante. On peut caractériser les études historiques de ce genre à partir de trois interrogations : sur la nature des sources exploitées, sur les questions posées à partir de ces sources, et sur la méthodologie mise en œuvre pour y parvenir ${ }^{10}$.

7 Au cours des décennies, les sources utilisées pour étudier la guerre se sont beaucoup élargies; voici une liste à titre d'exemple :

- Affiches gouvernementales

- Articles de la Presse nationale, régionale ou spécialisée

- Cartes postales illustrées

- Cartographie militaire, rapports militaires

- Chansons populaires des années de guerre-

- Cinéma des années de guerre

- Procès-verbaux de tribunaux, civils ou militaires

- Correspondance diplomatique

- Courrier des lecteurs de la presse

- Débats parlementaires 
- Dessins de presse

- Dossiers médicaux

- Enregistrements d'entretiens des soldats ou des civils après le conflit

- Journaux intimes de soldats ou de civils

- Lettres des soldats

- Littérature savante ou populaire des années de guerre

- Mémoires d'officiers

- Mémoires de militants antiguerre

- Mémoires de soldats de rang

- Mémoires des généraux et des dirigeants politiques

- Monuments aux morts

- Peinture et sculpture des années de guerre

- Photographie

- Programmes des concerts savants ou populaires

- Rapports gouvernementaux et ministériels

- Trouvailles des fouilles archéologiques de champs de bataille ou d'autres lieux historiques (camp de prisonniers...)

Cet élargissement considérable des types de sources historiques a accompagné une attention de plus en plus précise portée à la " grammaire » de ces sources, encouragée par la compréhension de l'importance des représentations mentales des aspects de la guerre. Ainsi, celui ou celle qui travaille sur les monuments tiendra compte du fait que les décisions les concernant furent prises non pas par les familles des victimes dont les noms y figurent, mais par l'élite municipale au nom de la communauté entière. Les modalités de la production des monuments constituent un champ d'étude important car elles permettent d'éclairer le sens social et politique de ces monuments. De façon similaire, le travail sur le cinéma des années de guerre n'a pas oublié d'analyser la musique qui accompagnait les bobines, partitions de morceaux recommandés pour chaque scène du film muet. Les conditions de réception des films sont donc prises en compte. Les archéologues des champs des batailles, quant à eux, ont mis en œuvre toutes les connaissances accumulées par les archéologues d'époques plus anciennes, afin de contextualiser les objets trouvés. Et moi-même, travaillant sur les chansons de music-hall dus chercher à comprendre les conditions de performance et notamment le poids de l'obligation de faire chanter le refrain d'une chanson à tout l'auditoire, obligation qui tendait à imposer des paroles consensuelles. ${ }^{11}$

\section{Trois configurations historiographiques concernant la Grande Guerre}

Antoine Prost et Jay Winter, dans leur livre Penser la Grande Guerre ${ }^{12}$, entreprennent une catégorisation des travaux historiques sur ce conflit en les divisant en trois grandes "configurations", nées consécutivement mais dont la nouvelle se rajoute aux précédentes sans les remplacer. D'ailleurs, les auteurs nous mettent en garde contre la supposition d'une domination exclusive d'une forme d'histoire à chaque période :

Si l'accent est mis d'abord sur l'histoire militaire et diplomatique, puis sur l'histoire sociale et enfin sur l'histoire culturelle, tous les types d'histoire sont présents au sein de 
chaque configuration: c'est leur place respective qui se modifie, leur poids qui change, leur rôle dans la problématique et l'argumentation qui se transforme ${ }^{13}$.

11 Nous le verrons, c'est le choix des questions privilégiées qui caractérise le plus sûrement ces configurations.

\section{Histoire diplomatique et militaire}

12 La première configuration est celle de l'histoire diplomatique et militaire, enclenchée dès la fin de la Première Guerre ${ }^{14}$. Parfois sponsorisée par les gouvernements, cette historiographie, très abondante, vise à expliquer, et souvent à justifier, les décisions diplomatiques et stratégiques des gouvernements et des généraux. Les sources exploitées sont les archives des instances gouvernementales, militaires et diplomatiques. Les questions posées sont celles de la stratégie des quartiers généraux militaires et diplomatiques. Les mémoires de guerre de David Lloyd George ${ }^{15}$, de Douglas Haig et d'autres jouissaient notamment d'une grande influence.

13 L'histoire militaire est passée par deux grandes périodes. Pendant les premières décennies, l'expérience du soldat de rang n'était pas considérée comme digne d'intérêt par l'historien. L'expérience du soldat pouvait être respectée par les étudiants de littérature, et les poètes anglais soldats de la Première Guerre faisaient déjà partie du canon littéraire, mais l'historien n'avait pas, pensait-on, à prendre cette parole en compte.

14 L'histoire militaire reste jusqu'à nos jours un composant majeur de l'historiographie. Selon Pierre Purseigle

L'histoire de la guerre est ... pleinement intégrée au paysage universitaire britannique ... Les principales universités britanniques ...emploient des historiens de la guerre et font une place variable mais significative à l'étude des opérations militaires en tant que telles. Ces dernières sont par ailleurs l'objet d'une attention toute particulière dans les académies militaires qui recrutent ... de nombreux historiens de formation universitaire. ${ }^{16}$

15 Mais cette histoire a changé de nature. Nous y trouvons de nos jours bien souvent l'expérience des soldats de rang. Sans doute l'avancée de l'esprit démocratique, le relâchement lent, mais sûr, de l'élitisme social rigide, l'expansion massive du nombre d'historiens pendant les "trente glorieuses", et l'amorce de la nouvelle histoire avec l'influence des historiens marxistes, ont participé au renversement des perspectives. L'extension massive de l'intérêt public pour des productions historiques - livres, revues, films ou documentaires télévisuels $-\mathrm{y}$ contribue également. Même des livres de plus de 700 pages tels que Tommy: the British Soldier on the Western Front du regretté Richard Holmes ${ }^{17}$ ont pu devenir des best-sellers.

Dans ces histoires sur la vie des soldats, on trouve des textes qui ont transformé notre compréhension de la guerre, dont nous ne citerons ici que quelques exemples. Dans Trench Warfare 1914-1918, The Live and Let Live System, de Tony Ashworth ${ }^{18}$, l'historien prend le pari d'étudier la vie des soldats en dehors des temps de batailles, et découvre un système informel mais omniprésent de "vivre et laisser vivre " par lequel les soldats tentèrent de rendre moins dangereuse la guerre en se mettant d'accord avec l'ennemi pour éviter de se battre. Un exemple suffira: la ritualisation des bombardements, ce qui signifiait que les troupes ennemis se mettaient d'accord clandestinement pour bombarder chacun l'autre à des endroits et des heures communiqués d'avance. Ainsi il y avait peu de 
pertes humaines, mais les soldats de rang pouvaient justifier auprès de leurs hiérarchies respectives qu'ils avaient envoyé le nombre d'obus prévu.

Une autre contribution originale est celle d'Ilana Bet-El, qui part du constat du mythe national d'une armée de joyeux volontaires impérialistes, afin d'étudier un groupe souvent oublié, les conscrits : ceux qui ont refusé de s'engager volontairement et y ont été contraints par les lois de $1916^{19}$. Il s'agit de la majorité des combattants britanniques de la Grande Guerre. Le livre de Richard Van Emden sur les prisonniers de guerre essaie également de communiquer l'expérience d'un groupe ignoré par une historiographie nationale de la victoire ${ }^{20}$.

L'histoire universitaire va beaucoup se diversifier après les années 1960, et dans les universités l'histoire militaire ne gardera pas sa position dominante. Ceci n'est pas le cas en ce qui concerne la production historique plus large. Même au XXIe siècle, du point de vue de l'édition, l'histoire militaire tient le haut du pavé. Des ouvrages tels que The Soldier's War de Richard Van Emden ${ }^{21}$, ou Bloody Victory de l'universitaire William Philpott ${ }^{22}$ rencontrent un vif succès. Et si ce dernier ouvrage contient un chapitre sur l'expérience des civils en Angleterre, et un autre sur le souvenir et la commémoration, le reste de ses 700 pages est consacré à l'histoire des batailles.

Les très nombreux mémoires ou journaux intimes se vendent bien également. Ces mémoires, ou parfois des recueils de lettres, sont d'une grande richesse. Il s'agit souvent d'officiers ${ }^{23}$, toutefois les soldats de rang sont de mieux en mieux représentés. Il peut s'agir de soldats professionnels ${ }^{24}$ ou de soldats volontaires ${ }^{25}$. Les récits des conscrits complètent le tableau ${ }^{26}$. Des recueils d'histoire orale ont également été produits, comme la série « Forgotten Voices » éditée en collaboration avec l'Imperial War Museum, principal musée militaire du pays, connu pour sa muséographie moderne et son ouverture d'esprit. ${ }^{27}$ Cette série vise à retracer le vécu des soldats à travers l'histoire orale ${ }^{28}$, basée sur des entretiens organisés depuis les années 1970. La série traite de plusieurs guerres du XXe siècle.

La prise en compte de l'expérience individuelle des soldats n'empêche pas toujours la présentation d'un version héroïque de la guerre. Une série, d'une dizaine de livres, «VCs of the First World» cherche à mettre en avant «l'héroïsme » des soldats anglais, en racontant l'histoire de chaque soldat qui a reçu la Victoria Cross durant la guerre ${ }^{29}$.

21 L'histoire militaire, si elle a évolué, est donc bien vivante. L'histoire diplomatique, au contraire, attire de nos jours nettement moins d'intérêt. L'arrivée du centenaire, pourtant, a remis à l'ordre du jour la question des causes immédiates de la Grande Guerre, question qui relève pour beaucoup d'auteurs de l'histoire diplomatique ${ }^{30}$. L'ouvrage de Christopher Clark est devenu l'ouvrage d'histoire diplomatique le plus vendu depuis bien longtemps. ${ }^{31}$ Son point de vue, selon lequel aucun pays ne porte une responsabilité écrasante dans le déclenchement du conflit, a contribué à faire de son livre un best-seller en Allemagne.

\section{Histoire sociale et politique}

22 La deuxième configuration qu'évoquent Prost et Winter est née pendant les "trente glorieuses » avec la montée de l'histoire sociale, portée par une avancée de l'esprit démocratique, par une démocratisation de l'accès aux études supérieures et par une certaine influence du marxisme au sein des universités ${ }^{32}$. Elle produit encore aujourd'hui 
un nombre important d'écrits. On s'intéresse aux civils (même si on les définit souvent par rapport aux projets impériaux, en tant que « front intérieur »). Plus généralement, on cherche à évaluer l'expérience d'autres groupes que les combattants : les infirmières ${ }^{33}$, les pacifistes $^{34}$, les prêtres ${ }^{35}$. Une conscience de la concentration massive de l'historiographie sur l'expérience des hommes dans la guerre a provoqué des tentatives de synthétiser l'expérience des femmes, souvent d'un point de vue féministe ${ }^{36}$. Ces tentatives restent pourtant peu nombreuses, et bien des aspects de la vie des femmes pendant le conflit restent inexplorés.

D'autres ont écrit l'histoire des organisations politiques ${ }^{37}$ et il existe une monographie sur le parti conservateur, mais les partis politiques ont généralement attiré peu d'intérêt. Pourtant l'union sacrée a bouleversé le fonctionnement des partis et il y a certainement matière à faire réfléchir...

L'histoire sociale qui étudie les siècles précédents a travaillé avec un grand intérêt sur le mouvement ouvrier et les syndicats. Etrangement, il n'y a, dans l'historiographie de la Première Guerre, quasiment aucune étude sur les syndicats. Pourtant, le nombre de syndiqués augmenta massivement et des grèves de masse firent partie du paysage social de 1916-1919. En 1918, il y eut même une grève historique de la police de Londres. Il existe une monographie comparatiste de John Horne, bien isolée, ${ }^{38}$ et deux ouvrages récents ont traité de l'industrie et des ouvriers, mais en se référant très peu ou pas du tout aux activités syndicales. ${ }^{39}$ Lorsque l'on fait une recherche dans le catalogue de la British Library sur les mots clé «First World War trade unions» ou «First World War trades unions", on reçoit dans les deux cas le conseil «Did you mean 'First World War trenches'?»! La bibliothèque ne trouve aucune monographie sur la question du syndicalisme britannique pendant la Grande guerre ${ }^{40}$. L'explication de cette importante omission n'est pas chose aisée, mais il est concevable que cette période où les syndicats furent dominés par une idéologie patriotique, ait moins attiré des spécialistes du syndicalisme que ne l'ont fait d'autres périodes.

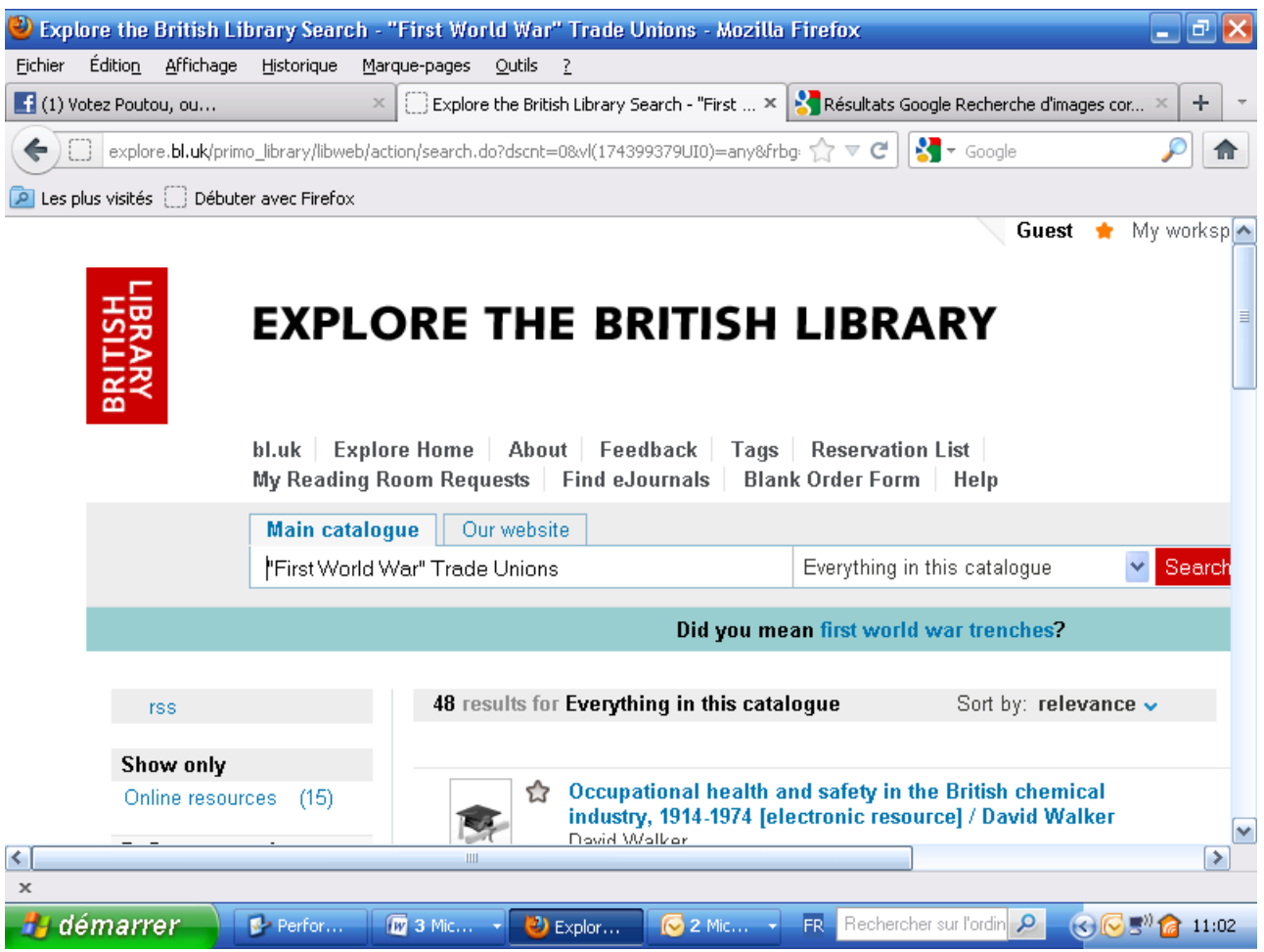


ution, pendant les «trente glorieuses » vers l'histoire sociale fut générale, quelle que soit la période étudiée. Les études de la Première Guerre ne font pas exception. On peut citer à cet égard le texte fondateur d'Arthur Marwick, The Deluge, qui se concentre sur la société civile ${ }^{41}$, comprenant des chapitres tels que "Changed Men", "New Women » et «After the Deluge ». Le livre d'A. J. P. Taylor, très influent pendant plusieurs décennies, porte beaucoup d'attention à l'histoire sociale ${ }^{42}$, et un historien de la génération suivante, $\mathrm{J}$. M. Bourne, consacre une grande partie de son livre à ce sujet ${ }^{43}$. Lorsque Trevor Royle, en 2006, écrit sa monographie sur l'Ecosse de la Grande Guerre, les titres des chapitres comprennent «The Workshop of War », «Women's work» et « Red Clydeside and opposing Armageddon ». Dans l'histoire orale, il existe également quelques contributions ${ }^{44}$. Le projet de l'Imperial War Museum, lancé pour le centenaire, «Lives of the First World War ", qui vise une présentation d'un très grand nombre de documents personnels (lettres, journaux intimes, photos...) incluent explicitement les civils dans son appel à contribution. ${ }^{45} \mathrm{Il}$ faut convenir, néanmoins, que le "tournant social», dans l'étude de la Première Guerre, a été moins marqué que dans l'étude d'autres périodes historiques telles que la période victorienne.

\section{Histoire culturelle}

La troisième grande configuration historiographique définie par Prost et Winter est celle de l'histoire culturelle, qui tient une position dominante à partir des années 1980 ou 1990 46. Pas toujours facile à définir, cette histoire incarne un intérêt particulier pour la représentation de la guerre, pour la construction des discours sur la guerre, pour des questions d'identité (représentation de soi, donc) etc. Sa montée en puissance ${ }^{47}$ s'est $^{\text {s. }}$ déroulé parallèlement à une réduction de l'influence de théories matérialistes sur l'histoire, ou d'un rejet des "grands récits» notamment celui du marxisme (généralement citée dans sa version stalinienne) ${ }^{48}$. Pour les historiens universitaires, l'histoire culturelle pouvait représenter à la fois une possibilité de se rapprocher d'autres disciplines (critique littéraire, études culturelles ...), et peut-être aussi une position de compromis qui permettait de continuer à privilégier l'histoire de ceux d'en bas, sans s'engager à mettre en œuvre une analyse d'histoire sociale à forte connotation politique. Néanmoins, le choix de certaines maisons d'édition comme Cambridge University Press de publier des collections d'ouvrages sous le titre général "Studies in the social and cultural history of modern warfare $»^{49}$ là où d'autres, telles que Manchester University Press ont choisi comme titre de collection «Cultural History of Modern War $»^{50}$ témoigne de la frontière floue entre ces deux configurations.

Certaines théories postmodernistes (rarement issues directement d'historiens) ont prétendu qu'il était impossible de retrouver la vérité d'une période historique, l'ensemble de nos connaissances se réduisant à une analyse de différents discours et représentations ${ }^{51}$. Le livre de Keith Jenkin ${ }^{52}$ représente bien ce courant de pensée :

$\mathrm{Carr}^{53}$ and Elton ${ }^{54}$ are too committed to the pretence that they can engage in a 'real' dialogue with the 'reality' of a (somehow) non-historiographically-constituted-pastas-history. ${ }^{55}$

In fact, 'history' now appears to be just one more 'expression' in a world of postmodern expressions: which of course is what it is. ${ }^{56}$

Si les versions les plus tranchées de ces théories rendraient impossible ou futile la tâche de l'historien, l'accent renforcé mis sur le discours et la représentation a permis un enrichissement du travail historique, et l'histoire culturelle a pu s'imposer. Lorsqu'Ilana

Revue Française de Civilisation Britannique, XX-1 | 2015 
Bet-El écrit sur les conscrits, à la fin des années 1990, elle ne se limite pas à une description de leur situation matérielle, mais passe beaucoup de temps sur leurs représentations de la vie militaire et de leur propre place. L'effet des mythes d'héroïsme est étudié, ainsi que la question de savoir à quel point le conscrit se voit comme militaire ${ }^{57}$ . Dans l'ouvrage de Rachel Duffet sur les soldats et la nourriture ${ }^{58}$, le sens de la nourriture pour les soldats est étudié, non pas seulement la logistique et l'importance stratégique. Dans un sens l'histoire culturelle est une nouvelle influence généralisée au sein de l'historiographie, plutôt qu'une catégorie étanche d'écrits historiques.

Un des ouvrages fondateurs de l'histoire culturelle sur la Grande Guerre fut celui de Paul Fussell ${ }^{59}$, qui explore les représentations du conflit dans les œuvres littéraires, et réfléchit sur les similitudes de l'autobiographie et de l'œuvre de fiction. Il défend l'utilisation d'œuvres littéraires comme source pour écrire de l'histoire, et cette approche deviendra courante au cours des années 1970 et 1980.

Une des forces reconnues de l'histoire culturelle de la Première Guerre est l'importance qui est donnée au vécu individuel ; l'histoire nous intéresse car nous sommes humains, et l'aspect humain, au-delà de généralisations sur les classes sociales ou forces politiques, doit transparaître. Les critiques principales de ce genre d'histoire ont été que les institutions (l'Etat, l'armée, les partis politiques, les syndicats etc.) peuvent disparaitre de l'analyse, ne laissant que des individus désocialisés et bardés d'une "culture " parfois supposée être la même pour tous les individus d'une société. La priorité croissante au sein de l'histoire culturelle donnée à l'histoire des représentations (donc une histoire plurielle) peut pallier partiellement à ce dernier écueil. D'ailleurs, des étiquettes précédentes (telles que «l'histoire des mentalités ») ont tendance à s'effacer devant celle de « l'histoire des représentations ${ }^{60}$. "

Le dernier thème qui monte en puissance depuis 20 ans au sein de l'histoire culturelle en Grande-Bretagne semble être justement celui de la commémoration, de la représentation du passé donc. ${ }^{61}$ Nous verrons qu'un grand nombre de jeunes chercheurs y travaillent. La réflexion ouvert en France par Pierre Nora et d'autres ${ }^{62}$ 'est pas étrangère à la popularité de ce nouveau thème.

\section{Controverses historiographiques}

Depuis une décennie, on perçoit un nouveau phénomène dans l'historiographie britannique : le révisionnisme. Certains historiens ont choisi l'étiquette "révisionniste " pour décrire leur tentative sophistiquée de rouvrir certaines questions qu'ils trouvaient mal exploitées. ${ }^{63}$ Le phénomène n'est pas sans lien avec l'influence grandissante des idées néolibérales dans l'économie, et le recul des idéologies de gauche au sein de l'université et ailleurs. L'historien révisionniste affirme qu'un consensus pacifiste et excessivement critique de la hiérarchie militaire domine le pays depuis les années 1960. Ils citent généralement la comédie musicale «Ah Dieu que la guerre est jolie! », la série télévisée «Blackadder » ou le livre d'Alan Clark «The Donkeys $»^{64}$ comme des exemples, voire des causes, d'une vision erronée de la guerre. S'opposant à la conception de la guerre comme une entreprise impérialiste peu démocratique et caractérisée par une hiérarchie sociale rigide, les révisionnistes défendent généralement la conception selon laquelle la hiérarchie militaire a fait du mieux qu'elle pouvait, au vu des connaissances de l'époque, et que leurs capacités militaires étaient d'un très haut niveau. De nombreux livres ont entrepris de défendre les généraux. Dans certains cas on peut soupçonner des auteurs, 
salariés de l'institution militaire, de plaidoyers pro domo. Sans doute le plus virulent est Gordon Corrigan ${ }^{65}$, ancien officier de l'armée. Niall Ferguson ${ }^{66}$ est un autre historien révisionniste dont les écrits ont eu un énorme écho. Le nouveau livre de William Philpott, Bloody Victory, qui soutient qu'il faut trouver un équilibre entre l'étude de la société et l'étude des batailles, appartient aussi au camp révisionniste. Bien que ce groupe ne soit pas nombreux, il jouit d'une impressionnante influence. Ainsi, Gary Sheffield ${ }^{67}$ a rédigé une bonne partie du site web "BBC History " concernant la Première Guerre, un site jouissant d'une réputation inégalable auprès du grand public ${ }^{68}$.

33 Le résultat des efforts de ces historiens est difficile à évaluer, mais ils ne semble pas tout à fait couronnés de succès. Un sondage de janvier 2014 révélait que $52 \%$ des sondés ${ }^{69}$ était d'accord avec la phrase

British troops in World War One were badly served by their generals, who made poor strategic decisions and needlessly wasted thousands of lives in pointless attacks.

Seuls $17 \%$ des sondés avaient une opinion positive des généraux. Il est intéressant de noter que cette mauvaise réputation des généraux dépend peu des opinions politiques des sondés : $50 \%$ des électeurs conservateurs et $55 \%$ des électeurs travaillistes partageaient cette vision négative. Il se peut que la mémoire familiale et locale soient plus fortes que les ouvrages largement commentés des révisionnistes, et bien des gens furent plus impressionnés par les déclarations du dernier soldat de la Grande Guerre, Harry Patch, avant sa mort en 2009 :

When the war ended, I don't know if I was more relieved that we'd won or that I didn't have to go back. Passchendaele was a disastrous battle - thousands and thousands of young lives were lost. It makes me angry. Earlier this year, I went back to Ypres to shake the hand of Charles Kuentz, Germany's only surviving veteran from the war. It was emotional. He is 107. We've had 87 years to think what war is. To me, it's a licence to go out and murder. Why should the British government call me up and take me out to a battlefield to shoot a man I never knew, whose language I couldn't speak? All those lives lost for a war finished over a table. Now what is the sense in that ?"70

Les histoires révisionnistes ne représentent pas pour autant un retour à l'histoire militaire d'antan, ce qui ne serait plus concevable aujourd'hui. Elles appellent davantage à une relativisation des aspects choquants de la conduite de la guerre par la hiérarchie militaire.

\section{Les travauX universitaires du XXle siècle}

Les archives de thèses mises en ligne par la British Library ${ }^{71}$ donnent un aperçu de l'état actuel de l'historiographie universitaire britannique. Celles-ci ne sont pas exhaustives, mais viennent d'un grand nombre d'universités britanniques et constituent un échantillon représentatif. Exactement 103 thèses ont été soutenues entre 2000 et 2014 qui avaient comme thème principal « La Grande Guerre ».

Le sujet de la thèse est généralement choisi par le doctorant en consultation avec son directeur de recherche. Les choix des sujets reflètent les intérêts des historiens enseignant dans les universités, car c'est généralement en suivant les séminaires existants que le doctorant trouve une idée pour sa recherche. Il y a une large mesure de liberté intellectuelle, mais aussi des pressions formelles et informelles. Si le doctorant cherche à obtenir un poste à l'université, denrée bien rare, il vaut mieux qu'il mesure 
l'effet de toute approche controversée ou excessivement novatrice: les universités, comme toute institution, ont leurs conservatismes. Par ailleurs, une minorité de thèses est financée par l'institution militaire.

Regardons donc les 103 thèses, à la lumière de la catégorisation développée par Prost et Winter. À leurs trois configurations : histoire diplomatique et militaire, histoire sociale, et histoire culturelle, nous nous voyons contraints d'ajouter des sous-catégories.

\begin{tabular}{|l|c|c|}
\hline \multicolumn{2}{|c|}{ Base = 103 thèses soutenues dans des universités britanniques depuis 2000 } \\
\hline Catégorie & $\begin{array}{l}\text { Nombre de } \\
\text { thèses }\end{array}$ & $\begin{array}{l}\text { Pourcentage des } \\
\text { thèses }^{72}\end{array}$ \\
\hline $\begin{array}{l}\text { Histoire militaire et } \\
\text { diplomatique }{ }^{73} \text { dont }\end{array}$ & 25 & 24,3 \\
\hline Histoire militaire & 19 & 18,4 \\
\hline Histoire de la diplomatie & 6 & 5,8 \\
\hline $\begin{array}{l}\text { Histoire sociale et politique, } \\
\text { dont }\end{array}$ & 32 & 31,0 \\
\hline Histoire politique & 6 & 5,8 \\
\hline Histoire sociale générale & 16 & 15,5 \\
\hline Histoire médicale & 6 & 5,8 \\
\hline Histoire culturelle, dont & 46 & 44,7 \\
\hline Études du genre & 4 & 3,9 \\
\hline Histoire culturelle générale & 16 & 15,5 \\
\hline Lesécrits de femmes & 6 & 5,8 \\
\hline Histoire de la culture populaire & 6 & 5,8 \\
\hline Histoire et commémoration & 13 & 12,6 \\
\hline & & \\
\hline
\end{tabular}

L'histoire militaire et diplomatique reste bien présente. ${ }^{74}$ Une série de titres traitent de stratégie et de tactique militaire : le rôle de la cartographie, la politique d'utilisation des chars d'assaut, la part jouée par la cavalerie ou les rangs intermédiaires de la hiérarchie de l'armée. Quatre autres thèses se penchent sur un régiment particulier et l'histoire de sa guerre. Pour les régiments recrutés dans une région géographique restreinte, certains auteurs étudient le lien entre le régiment et sa ville d'origine. Enfin, une thèse revient en détail sur les exécutions militaires durant la guerre ${ }^{75}$.

Six ouvrages examinent les discours et les conceptions des diplomates : la compréhension $\mathrm{du}$ Califat dans les milieux gouvernementaux britanniques, la politique des Pays-Bas neutres, l'attitude de l'État britannique envers le sionisme, par exemple. On remarquera que les thèses s'articulent autour des représentations et des discours: l'influence de l'histoire culturelle dans son sens le plus large est apparente.

41 L'histoire militaire vue d'en bas (la vie des soldats ordinaires, des infirmières ou des marins du rang) est presque absente. Grand succès de l'édition, elle a très peu sa place dans l'historiographie universitaire. On peut le regretter, car les centaines de recueils de récits, lettres, journaux intimes ou entretiens qui décrivent la vie militaire vue d'en bas souffrent souvent d'un manque de théorisation, alors que la production universitaire qui 
aurait pu fournir cette théorisation semble ignorer le thème. On peut penser que, malgré les progrès énormes faits dans l'écriture de l'histoire vue d'en bas, sur un sujet si important pour l'histoire nationale officielle, la domination de la vision d'en haut reste franche et massive. Seules deux thèses relèvent directement de la vie des soldats du rang:

A war unimagined; Food and the rank and file soldiers of the First World War, Duffett, Rachel, University of Essex, 2009.

The chances of survival: personal risk assessment and attitudes to death among German and British soldiers in the Great War, 1914 1918, Watson, Alexander, University of Oxford, 2005.

\section{L'histoire politique}

Une série de thèses explorent les discours politiques concernant la guerre, et le rôle d'organisations politiques ou d'institutions particulières telles que la Justice. La réaction des organisations féministes à la guerre intéresse un des auteurs, le parti conservateur est le thème d'un autre ouvrage, et un troisième est consacré au Parti conservateur dans une région précise (le Nord-Est du Pays de Galles). Ces thèses se basent sur un examen minutieux des archives disponibles. La " construction de l'identité » qui est devenue un thème majeur pour les historiens en général, est bien présente. Une histoire des liens entre le pacifisme et l'art moderne complète ce groupe.

\section{L'histoire sociale}

Nous pouvons ranger dans la catégorie, bien large, d'histoire sociale, une quinzaine de thèses. Elles cherchent à approfondir les connaissances dans des domaines particuliers (un groupe social peu étudié, ou la guerre dans une région donnée...), ou à exploiter un nouveau corpus. La communauté catholique, les enfants, les immigrés allemands à Glasgow, les peuples indigènes de l'empire, ainsi que les veuves de guerre, ont chacun leur chercheur. Il ne s'agit aucunement de simples récits : les analyses de l'identité ${ }^{76}$ et de discours idéologique ${ }^{77}$ sont bien présentes.

L'utilisation de nouvelles archives pas exploitées est illustrée par le travail de Sarah Pedersen, qui choisit une période plus longue que la guerre afin de confronter une réalité d'expression publique féminine à la vision générale que nous avons des contraintes qui pesaient sur les femmes de l'époque ${ }^{78}$.

\section{Histoire Médicale}

Un nombre surprenant de thèses (six) témoigne d'un domaine qui monte en puissance : "l'histoire médicale ", qui constitue sans doute une sous-catégorie de l'histoire sociale. L'avantage de ce domaine demeure dans le fait que les archives médicales sont bien fournies, et une grande partie a été conservée. Il a aussi l'avantage de traiter d'éléments relativement objectifs: des symptômes clairement constatés. Relativement, car la conception de la maladie mentale en particulier est en évolution constante depuis plus d'un siècle, et il n'y a pas de consensus même de nos jours. On voit que des conditions psychologiques constituent un thème de prédilection. Quatre thèses traitent du "Shell shock» (on dirait aujourd'hui "Syndrome de stress post-traumatique»). Ce sujet est étudié depuis plusieurs décennies ; ces auteurs étendent le champ de l'étude : une auteure étudie ces problèmes psychologiques chez les infirmières au front ${ }^{79}$, une autre traite des 
effets à long-terme de ces maladies ${ }^{80}$; une troisième incorpore une étude statistique de 500 cas de $«$ shell shock $»^{81}$.

\section{Questions de genre} populaire pendant la guerre, et les autres des représentations postérieures. La télévision ${ }^{86}$ , le cinéma ${ }^{87}$ et la littérature enfantine ${ }^{88}$ comptent un ouvrage chacun. La culture populaire n'est donc pas négligée, et la plainte de Peter Bailey en 1978 que l'étude des loisirs est absente du domaine de l'histoire n'est plus tout à fait justifiée ${ }^{89}$. L'expérience de guerre en tant que thème traité dans la culture est mise en avant; cependant, l'histoire des loisirs des années de guerre elles-mêmes est quasiment absente.

\section{Histoire et Commémoration}

51 La popularité du thème de la commémoration témoigne de priorités similaires. Plutôt qu'écrire sur l'expérience de la guerre, ces ouvrages se concentrent sur le processus, complexe et fascinant, de la transformation de l'histoire en mémoire. Treize thèses sont consacrées à la commémoration. Elles peuvent se focaliser sur des activités commémoratives sélectionnées ${ }^{90}$, sur la commémoration dans une région spécifique ${ }^{91}$, ou sur un thème présent dans les commémorations ${ }^{92}$. 
démarches caractérisent l'influence de l'histoire culturelle. Elles ne font pas consensus, et ont notamment été l'objet de critiques de la part d'historiens marxisants. Eric Hobsbawm exprimait ses réserves sur cette question lors d'un entretien en $2008^{93}$ :

The main trouble about the cultural turn is that an awful lot of it tends to move away not merely from the social element in history, but also from the real history. For instance, the enormous range of studies of memory in the 1970s and 1980s which are quite new, which didn't happen in our day. Well, memory is about today, memory isn't about what happened, it's about what people later on think happened.

PO: It's a completely different sort of source to a primary source from the time, of course?

EH: Either a primary source of even an attempt to reconstruct what really happened on the ground. I mean what people thought afterwards is very important, but it's important for the period in which they think and not for the period about which they think.

PO: Yes, I see the distinction that you're making there.

EH: That's the point. I mean the problem for instance today is the enormous amount about the memory of World War One. The question is, why have we rediscovered World War One, which is a long long time ago, and what does it mean. But whatever's written about the memory of the Battle of the Somme is not the same as actually-

PO: It actually concerns 21st century Britain more than it does 20th century Britain.

EH: That's exactly what I was saying, yes.

53 Cette critique ne s'applique pas pleinement aux thèses que nous citons ci-dessus, dans la mesure où plusieurs d'entre elles tentent de reconstruire le processus de commémoration dans une période révolue, mais il s'agit néanmoins, comme le souligne le Pr Hobsbawm, d'une histoire de perceptions qui peut avoir l'inconvénient de s'éloigner de l'histoire de l'activité humaine.

\section{Quelques absences}

Nous constatons que la production historique universitaire britannique est impressionnante et variée. Il est important de souligner que les différents thèmes sont distribués parmi toutes les universités - il n'y a pas de spécialisation évidente par établissement, ce qu'on peut considérer un point fort, qui peut permettre des échanges riches entre collègues d'un même établissement. Il y a pourtant quelques absences notables importantes. La vie au travail et la vie syndicale sont absentes, et les thèmes semblent très centrés autour de la Grande- Bretagne, à l'exception des deux thèses sur la commémoration du côté allemand. Les colonies britanniques sont absentes, y compris les plus importantes: l'approche "postcoloniale» ne semble pas bien influente dans ce domaine. Les historiographies universitaires sont restées résolument nationales dans chaque pays. En effet, en cherchant parmi les 160 thèses soutenues en France depuis 2002 ${ }^{94}$ et dont le résumé évoque la Première Guerre, seulement trois thèses, toutes comparatistes, mentionnent la Grande-Bretagne.

Malgré la quantité et la variété d'ouvrages produits, il existe donc des lacunes. En ce qui concerne l'histoire militaire, Pierre Purseigle a regretté l'absence d'études comparatistes 95 .

...les approches transnationales et comparées demeurent trop rares. Soucieux

d'explorer- si ce n'est de célébrer - l'exception britannique, le primat du cadre 
national obère finalement une meilleure compréhension de l'expérience

britannique du conflit. ${ }^{96}$ spécialistes de périodes bien plus longues - médiévalistes ou spécialistes du XIXe siècle, etc. Les historiens spécialistes de la Première Guerre se concentrent sur une période de quatre ans et demi ! Comment justifier d'un point de vue scientifique une telle myopie? En tout cas, cela entraîne parfois un manque de recul, et des continuités avec le monde de l'avant-guerre ou de l'après-guerre ne sont pas toujours relevées. Des études qui couvrent des périodes plus longues seraient indispensables. Mais le poids de la tradition et la formation des chercheurs rendra sans doute une telle évolution problématique. ${ }^{97}$

\section{Conclusions}

Voilà donc un tableau synthétique des écrits britanniques sur la Grande Guerre. On nous a fait remarquer qu'il est incomplet, et nous l'admettons volontiers. Notamment, nous n'avons pas couvert, par manque de connaissance, l'historiographie post-coloniale sur la guerre, produite en Inde et dans d'autres ex-colonies. C'est un champ qu'il faudra explorer. Nous n'avons pas voulu, non plus, nous étendre plus longuement sur la production littéraire et autobiographique - de Vera Brittain à Robert Graves en passant par Siegfried Sassoon - qui, tout comme les écrivains pacifistes étrangers tel qu'Henri Barbusse, ont énormément influencé notre image de la guerre .

Il reste un point à couvrir, la question de l'histoire comme forme de communication. L'historien n'est pas seulement quelqu'un qui découvre des vérités (qu'il ou elle espère significatives) sur le passé, c'est aussi quelqu'un qui s'efforce à les raconter, à ses étudiants, aux intéressés du grand public, ou plus largement encore. La capacité de bien écrire ou communiquer et de structurer un récit qui attire le lecteur fait partie du métier. ${ }^{98}$ La popularité croissante des émissions historiques de télévision a contribué à élargir le champ d'action des historiens. Certains, tels que Simon Schama, Niall Ferguson ou Max Hastings sont devenus des personnages médiatiques. Les nouvelles technologies d'internet ont contribué aussi à de nouveaux moyens de diffusion. Tout récemment, à part la large gamme d'émissions de la BBC disponibles en permanence sur son site web, les podcasts de l'Université d'Oxford concernant la Grande Guerre, et ceux de l'Imperial War Museum, ont touché un large public.

Mais même en nous limitant à notre sujet principal, les écrits, on constate certains choix nouveaux. Des histoires en forme de catalogue, telles que The History of the First World War in 100 objects de Peter Doyle, par exemple, ou la «counterfactual history » qui essaie d'évaluer ce qui serait arrivé, par exemple, si l'archiduc n'avait pas été tué en $1914 .{ }^{99}$ Nous n'avons pas fini de voir de l'innovation et de nouvelles explorations dans les écrits sur la Grande Guerre. Le centenaire aura donné un coup de fouet supplémentaire à une activité déjà intense et fascinante. 


\section{BIBLIOGRAPHIE}

Adie, Kate, Fighting on the Home Front: The Legacy of Women in World War One, Londres, Hodder and Stoughton, 2013.

Allingham, Henry et Goodwin Denis, Kitchener's Last Volunteer, Edimbourg, Mainstream Publishing, 2008.

Arthur, Max, Forgotten Voices of the Great War, Londres, Ebury, 2002.

Arthur, Max, Last Post - the Final Word from Our First World War Soldiers, Londres, Phoenix, 2005.

Arthur, Max, The Road Home - the Aftermath of the Great War Told by the Men and Women Who Survived It, Londres, Phoenix, 2010.

Ashworth, Tony, Trench Warfare 1914-1918, The Live and Let Live System, Londres, Pan, 1980.

Atkinson, Diane, Elsie \& Mairi Go to War, Two Extraordinary Women on the Western Front, Londres, Arrow, 2010.

Barrett, Duncan (dir.), The Reluctant Tommy, Ronald Skirth's Extraordinary Memoir of the First World War, Londres, Macmillan, 2010

Battarbee, Keith, « The Quest for Coherence and Discipline » in La Civilisation: objet, enjeux, méthodes, revue Babel N 9, 2004.

Beckett, Ian, Home Front 1914 to 1918: How Britain Survived the Great War, Londres, National Archives, 2006.

Bet-El, Ilana, Conscripts, Forgotten Men of the Great War, Stroud, Sutton, 1999.

Bourne, J. M., Britain and the Great War, Londres, Hodder \& Stoughton, 1989.

Boyd, W., With a Field Ambulance at Ypres, New York, G. H. Dorem, 1916.

Braybon, Gail et Summerfield, Penny, Out of the Cage, Women's Experience in Two World Wars, Londres, Pandora Press, 1987.

Braybon, Gail, Women Workers in the First World War, Londres, Croom Helm, 1981.

Brown, Malcolm et Seaton Shirley, Christmas Truce, Basingstoke, Pan Books, 1999.

Cameron, Stewart, A Very Unimportant officer, Londres, Hodder \& Stoughton, 2009.

Campion Vaughan, Edward, Some Desperate Glory, The Diary of a Young Officer, 1917, Londres, Pen \& Sword, 2010 (1981).

Carr, E.H., Qu'est-ce que l'histoire? (1961), Paris, 10-18, 1996

Cassar, George H., Kitchener, Architect of Victory, Londres, William Kimber, 1977.

Cate Haste, Keep the Home Fires Burning: Propaganda in the First World War, Londres, Allen Lane, 1977.

Churchill, Winston, The World Crisis 1911-1918, New York, Free Press, 2005 (1931, 1959).

Clark, Alan The Donkeys, Londres, Pimlico, 1991 (1961).

Clark, Christopher, The Sleepwalkers, How Europe Went to War in 1914, Londres, Allen Lane, 2012. 
Collins, L. C., Theatre at war 1914-1918, Basingstoke, Macmillan, 1998.

Corns, Catherine et Hughes-Wilson, John, Blindfold and Alone - British Military Executions in the Great War, Londres, Cassell, 2005.

Corrigan, Gordon, Mud Blood and Poppycock, Londres, Cassell, 2003.

Corrigan, Gordon, Sepoys in the Trenches: The Indian Corps on the Western Front, 1914-15, Londres, Spellmount, 2006.

Corvisy, Catherine-Emilie et Molinari, Véronique, Les femmes dans l'Angleterre victorienne et édouardienne: Entre sphère privée et sphère publique, Paris, L'Harmattan, 2008.

Cosens, M., Lloyd George's Munitions Girls, Londres, Hutchinson, 1916.

Crozier, Frank P., A Brass Hat in No Mans Land, Londres, Jonathan Cape, 1930.

Crozier, Frank P., The Men I killed, Londres, Michael Joseph, 1937.

Cummins Kennedy, Thomas, The Hound of Conscience: a History of the No-conscription Fellowship, 1914-1919, Fayetteville, University of Arkansas Press,1981.

Dakers, Caroline, The Countryside at War 1914-1918, Londres, Constable, 1987.

Daniels, Henry et Collé-Bak, Nathalie (dirs), 1916, La Grande-Bretagne en guerre, Nancy, Presses Universitaires de Nancy, 2007.

De Groot, Gerard J., Blighty: British Society in the Era of the Great War, Londres, Longman, 1996.

Delacroix, C., Dosse, F., Garcia, P., et Offenstadt, N., Historiographies 1 : Concepts et débats, Paris, Gallimard, 2010.

Delahaye, Claire et Ricard, Serge (dirs). : La Grande Guerre et le combat féministe, Paris, L'Harmattan, 2009.

Douglas, Roy, The Great War, 1914-1918: the Cartoonists' Vision, Londres, Routledge, 1995.

Duffy, Christopher, Through German Eyes: The British and the Somme, 1916, Londres, Phenix, 2007.

Dungan, Myles, They Shall Not Grow Old: Irish Soldiers and the Great War, Dublin, Four Courts Press, 1997.

Ellsworth-Jones, Will, We Will Not Fight...: The Untold Story of World War One's Conscientious Objectors, Londres, Aurum Press 2008.

Elton, G. R., The Practice of History (1967), Londres, Wiley-Blackwell, 2001.

Evans, Richard, In Defence of History, Londres, Granta, 2000.

Faulkner, Neil, « La Somme, une bataille pour l'empire et le profit » in Henry Daniels et Nathalie Collé-Bak, (dirs), 1916, La Grande-Bretagne en guerre, Nancy, Presses Universitaires de Nancy, 2007.

Fell, Alison S. et Hallett, Christine E., (dirs) First World War nursing: new perspectives, New York, Routledge, 2013.

Ferguson, Niall, Empire, How Britain Made the Modern World, Londres, Allen Lane, 2003.

Ferguson, Niall, The Pity of War, Londres, Allen Lane, 1998.

Fuller, J. F. G., Troop Morale and Popular Culture in the British and Dominion Armies 1914-1918, Oxford, Clarendon, 1990.

Fussell, Paul, The Great War and Modern Memory, Oxford, Oxford University Press, 2000.

Gliddon, Gerald, VCs of the First World War: 1914, Stroud, Sutton, 1994 . 
Gliddon, Gerald, VCs of the First World War: Spring Offensive 1918, Stroud, Sutton, 1997.

Gliddon, Gerald, VCs of the First World War: Arras \& Messines, 1917, Stroud, Sutton, 1998.

Graves, Robert, Goodbye to All That, Londres, Penguin, 2000 (1957).

Grayzel, Susan R., Women and the First World War, Harlow, Longman, 2002.

Gregory, Adrian, The Last Great War: British Society and the First World War, Cambridge, Cambridge University Press, 2008.

Grieves, Keith, The Politics of Manpower 1914-1918, Manchester, Manchester University Press, 1988.

Hastings, Max, Catastrophe, Europe Goes to War 1914, Londres, William Collins, 2013.

Hermon, E.W. et Nason, Anne, For Love and Courage, Letters Home from the Western Front, Londres, Preface, 2008.

Higonnet, Margaret R., et al., (dirs) Behind the Lines: Gender and the Two World Wars, New Haven, Yale University Press, 1987.

Hobsbawm, Eric, « From Social History to the History of Society », Daedalus, vol. 100, No. 1, hiver 1971.

Holmes, R., Tommy -The British soldier on the Western Front 1914-1918, Londres, Harper Perennial, 2005.

Jenkins, Keith On 'What is history?' From Carr and Elton to Rorty and White, Abingdon, Routledge,1995. Junger, Ernst, Storm of Steel, nouvelle édition, Harmondsworth, Penguin, 2004

Keegan John, The First World War, Londres, Random House, 1998.

Keohane, Nigel, The Party of Patriotism: the Conservative Party and the First World War, Farnham, Ashgate, 2010.

Keren Michael et Herwig, Holger, War Memory and Popular Culture: Essays on Modes of Remembrance and Commemoration, Jefferson, Macfarland, 2009.

Lamin, Bill, Letters from the Trenches, A Soldier of the Great War, Londres, Michael O'Mara, 2009

Lebow, Richard, Archduke Franz Ferdinand Lives ! : A World without World War I, Londres, Palgrave Macmillan, 2014

Lee, Arthur Gould, No Parachute: A Fighter Pilot in World War I, Londres, Jarrolds, 1968.

Levine, Joshua, Forgotten voices of Dunkirk, Londres, Ebury, 2010.

Levine, Joshua, Forgotten voices of the Blitz and the battle for Britain, Londres, Ebury, 2006.

Levine, Joshua, Forgotten Voices of the Somme, Londres, Ebury, 2008.

Livingstone, Thomas, Tommy's War: the Diary of a Wartime Nobody, Londres, Harper Press, 2008.

Lloyd George, David, War Memoirs of David Lloyd George, (7 tomes), Londres, Oldham, 1936.

Macdonald, Lyn, 1914: The Days of Hope, Londres, Penguin, 1989.

Macdonald, Lyn, 1915: The Death of Innocence, Londres, Penguin, 1993.

Macdonald, Lyn, The Roses of No Man's Land, Londres, Penguin, 1993 (1980).

Macdonald, Lyn, To the Last Man: Spring 1918, Londres, Penguin, 1999.

MacManners, Hugh, Forgotten voices of the Falklands, Londres, Ebury, 2007. 
Macmillan, Margaret, The War that Ended Peace: How Europe Abandoned Peace for the First World War, Londres, Profile Books, 2013.

Marwick, Arthur, The Deluge, British Society and the First World War, Londres, Bodley Head, 1965

Marwick, Arthur, Total War and Historical Change: Europe 1914-55, Buckingham, Open University Press, 2001.

McLean, Iain, The Legend of Red Clydeside, Edimbourg, Donald, 1983.

Morrow, John H., The Great War - an Imperial History, Londres, Routledge, 2004.

Moynihan, Michael, God on Our Side -the British Padre in World War One, Londres, Secker and Warburg, 1983.

Omissi, David E., Indian Voices of the Great War: Soldiers' Letters, 1914-18, Basingstoke, Macmillan, 1999.

Oram, Gerard, « What alternative punishment is there? »: military executions during World War I, Milton Keynes, The Open University, 2000.

Paice, Edward, Tip and Run: the Untold Tragedy of the Great War in Africa, Londres, Phoenix, 2008.

Pankhurst, Sylvia, The Home Front, a Mirror to Life in England during the World War, Londres, Hutchinson, 1932.

Patch, Harry, The Last Fighting Tommy, Londres, Bloomsbury, 2008.

Philpott, William, Bloody Victory, the Sacrifice on the Somme, Londres, Abacus, 2010.

Poirrier, Philippe, Les enjeux de l'histoire culturelle, Paris, Editions du Seuil, 2004.

Priestley, J. B., Margin Released, Londres, Heinemann, 1962.

Prost, Antoine et Winter, Jay, Penser la Grande Guerre, Paris, Editions du Seuil, 2004.

Purseigle, Pierre, » Ecrire l'histoire du Déluge. Histoire et expérience britannique de la Grande Guerre » in Histoire@politique. Politique, Culture, Société, № 22, janvier-avril 2014.

Richards, Frank, Old Soldiers Never Die, Uckfield, Naval and Military Press, s.d

Robb, George, British Culture and the First World War, Basingstoke, Palgrave, 2002.

Rosmer, Alfred, Le Mouvement ouvrier pendant la guerre, tome 1, De l'union sacrée à Zimmerwald, Paris, Librairie du travail, 1936.

Rosmer, Alfred, Le mouvement ouvrier pendant la 1re guerre mondiale, tome 2, De Zimmerwald à la Révolution russe, Paris, Mouton et Cie, 1959.

Rowbotham, Edward, Mud, Blood and Bullets, Memoirs of a Machine Gunner on the Western Front, Stroud, Spellmount, 2010.

Royle, Trevor, The Flowers of the Forest - Scotland and the First World War, Edimbourg, Birlinn, 2007.

Rubin, Gerry, War, law and labour: the Munitions Acts, state regulation and the unions, 1915-1921, Oxford, Clarendon Press, 1987

Sheffield, Gary, Forgotten Victory - the First World War, Myths and Realities, Londres, Review Press, 2001

Sheffield, Gary, Leadership in the Trenches: Officer-Man Relations, Morale and Discipline in the British Army in the era of the First World War, Londres, Macmillan, 2000.

Sheffield, Gary, The Chief: Douglas Haig and the British Army, Londres, Aurum, 2011. 
Silbey, David, The British Working Class and Enthusiasm for War, 1914-1916, Londres, Frank Cass, 2005. Smith, Lyn, Forgotten voices of the Holocaust, Londres, Ebury, 2005.

Snape, Michael et Madigan, Edward (dirs), The Clergy in Khaki: New Perspectives on British Army Chaplaincy in the First World War, Farnham, Ashgate, 2013.

Snelling, Stephen VCs of the First World War: The Naval VCs, Stroud, Sutton, 2002.

Snelling, Stephen, VCs of the First World War: Gallipoli, Stroud , History, 2010.

Switzer, Catherine, Unionists and Great War Commemoration in the North of Ireland, 1914-1939, Dublin, Irish Academic Press, 2007

Taylor, A. J. P., The First World War - an Illustrated History, Harmondsworth, Penguin, 1966.

Terraine, John, Douglas Haig The Educated Soldier, Londres, Hutchinson, 1963.

Turner, William, The Accrington Pals, Preston, Lancashire County Books, 2000.

Van Emden, Richard et Humphries, Steve, All Quiet on the Home Front, an Oral History of Life in Britain during the First World War, Londres, Headline, 2003.

Van Emden, Richard, Boy soldiers of the Great War, Londres, Headline, 2005.

Van Emden, Richard, Britain's Last Tommies, Londres, Abacus, 2005.

Van Emden, Richard, Prisoners of the Kaiser, Barnsley, Pen \& Sword, 2000.

Van Emden, Richard, Sapper Martin, The Secret Great War Diary of Jack Martin, Londres, Bloomsbury, 2010.

Van Emden, Richard, The Soldiers' War: the Great War through Veterans' Eyes, Londres, Bloomsbury, 2008.

Waites, Bernard, A Class Society at War: England 1914-18, Leamington Spa, Berg, 1987.

Warner, Philip, Passchendaele, Londres, Pen \& Sword, 2005.

Wilkinson, Alan, The Church of England and the First World War, Londres, SCM Press, 1996.

Will Ellsworth-Jones, Will, We Will Not Fight...: The Untold Story of World War One's Conscientious Objectors, Londres, Aurum Press 2008.

Winter, Jay Murray, The Great War and the British People, Cambridge Massachussets, Harvard University Press, 1986.

Woods, Mike et Platts, Tricia, Bradford in the Great War, Stroud, Sutton, 2007.

\section{ANNEXES}

Annexe : les thèses en lien avec la Première Guerre mondiale soutenues dans des universités britanniques depuis 2000 .

Histoire militaire et diplomatique

Discipline in the Russian army in the First World War, Simmons, Paul, Oxford University, 2011 
War aims and peace conditions: Austro-Hugarian foreign policy in the Balkans, July 1914 May 1917, Fried, Marvin Benjamin, London School of Economics and Political Science (University of London), 2011

The German army on the Western Front, 1914-1918,Sheldon, John Aitken, University of Westminster, 2011

British strategy and oil, 1914-1923,Gibson, Martin William, University of Glasgow, 2012

An analysis and evaluation of British, French and German military field survey and mapping in the First World War: which country produced the best survey and mapping of the Western Front?, Chasseaud, Peter Hugh Lawrence, University of Greenwich, 2004.

The operational role of British corps command on the Western Front, 1914-18, Simpson, Andrew, University College London, 2001.

The theory and practice of tank co-operation with other arms on the western front during the First World War, Hammond, Christopher Brynley, University of Birmingham, 2005.

British Cavalry on the Western Front 1916-1918, Kenyon, David, University of Cranfield, 2008.

The British Infantry Officer on the Western Front, in the First World War: with special reference to the Royal Warwickshire Regiment, Kang, Changboo, University of Birmingham, 2007.

British generalship on the Western Front in the First World War, 1914-1918, Robbins, Simon Nicholas, King's College London, 2001.

British scientists and soldiers in the First World War (with special reference to ballistics and chemical warfare), David, Thomas Rhodri Vivian, Imperial College London, 2009.

The 1/6th and 1/10th Battalions of the King's (Liverpool) Regiment in the period of the First World War, McCartney, Helen B. University of Cambridge 2000

The 1/7't'h Battalion King's Liverpool Regiment and the Great War: the experience of a Territorial battalion and its home towns, Gregson, Adrian S., Coventry University, 2004.

The 51st (Highland) Division during the First World War, French, Craig F., University of Glasgow, 2006.

The 52nd (lowland) division in the Great War, 1914-1918, Forrest, Christopher S., The University of Salford, 2009.

International law at sea, Economic Warfare, and Britain's Response to the German U-boat Campaign during the First World War, Russell, Bruce, Open University, 2007.

The strength of an army: Ottoman military effectiveness in the First World War, Erickson, Edward J., University of Leeds, 2005.

"What alternative punishment is there?": military executions during World War I Oram, Gerard Christopher, The Open University, 2000.

Jihad made in Germany: Ottoman and German propaganda and intelligence operations in the First World War, Ludke, Tilman, Oxford University, 2001. 
The idea of an Arab caliphate in British Middle Eastern policy in the era of the Great War, Cox, Stephen Thomas, Durham University, 2003.

Guarded Neutrality: The internment of foreign military personnel in the Netherlands during the First World War, Wolf, Susan, University of Sheffield, 2008

Nationalism, discourse and imagination: British policy towards the Zionist movement during the First World War, Renton, James, University College London, 2003.

A war unimagined; Food and the rank and file soldiers of the First World War, Duffett, Rachel, University of Essex, 2009.

The chances of survival: personal risk assessment and attitudes to death among German and British soldiers in the Great War, 1914 1918, Watson, Alexander, University of Oxford, 2005.

The conceptual origins of the control of the air: British military and naval aviation, 1911-1918, Pugh, James Neil, University of Birmingham, 2013

The invasion question: Admiralty plans to defend the British Isles, 1888-1918, MorganOwen, David Gethin, University of Exeter, 2013

British infantry battalion commanders in the First World War, Hodgkinson, Peter Eric, University of Birmingham, 2014

Histoire politique et sociale

Manufacturing and the Great War, Osborne, Wayne D., Loughborough University, 2013

Shetland and the Great War, Riddell, Linda Katherine, University of Edinburgh, 2012

Citizens at war: the experience of the Great War in Essex, 1914-1918, Halifax, Stuart, University of Oxford, 2010

Above the battlefield: art for art's sake and pacifism in the First World War, Brockington, Grace, University of Oxford, 2003.

County Armagh and the Great War, 1914-1919, Cousins, J. C, Queen's University Belfast, 2010.

Feminism and the challenge of war: responses of the British Women's Suffrage Movement to the Great War, Lefebvre, Marc Andre Louis Alexis, Goldsmiths College London, 2009.

The Conservative party in north-east Wales, 1906-1924, Williams, Thomas Wyn, University of Liverpool, 2008.

The Unionist party and the First World War, Keohane, Nigel Thomas, Queen Mary College, 2005.

Property, liberty and obligation: the judicial role in the Great War. Foxton, David Andrew. , King's College London (University of London), 2001

Aspects of the great war in Carmarthenshire, Barlow, Robin, University of Wales, Trinity Saint David, 2000.

The West Country and the First World War: Recruits and Identities, Gale, Andy, Lancaster University, 2010.

Ireland's revolutionary war?: nationalist propaganda, the Great War, and the construction of Irish identity. Novick, Benjamin Zvi., University of Oxford, 2000. 
Enabling the Great War: Ex-Servicemen, the Mixed Economy of Welfare and the Social Construction of Disability, 1899-1930, Kowalsky, Meaghan Melissa Marie, University of Leeds, 2007.

Within their sphere? Women's correspondence to Aberdeen daily newspapers, 1900-1918, Pedersen, Sarah, Robert Gordon University, 2004.

Migrants and internees: Germans in Glasgow, 1864-1918, Manz, Stefan, University of Durham, 2001

The children's war: British children's experience of the Great War, Kennedy, Rosalind Joan Sarah, Goldsmiths College (University of London), 2006.

'Pitied but distrusted': discourses surrounding British widows of the First World War, Smith, Angela, University of Sunderland, 2007.

All the King's Men: Indigenous Peoples of the Dominions and the First World War, Winegard, Timothy C., Oxford University, 2010.

British Catholic identity during the First World War: the challenge of universality and particularity, Finlay, Katherine, University of Oxford, 2004.

The First World War and Voluntary Recruitment: A Forum for Regional Identity? An analysis of the nature, expression and significance of regional identity in Hull 1900-1916, Townsley, Helen, University of Sussex, 2007.

Death, Service and Citizenship in Britain in the First World War, Hughes, Anne Marie Claire, University of Manchester, 2010.

Problems, politics and personalities in the treatment of mental and nervous casualties in the British Army 1914-1918. Incorporating a statistical and analytical study of 500 case histories, Hopkins, John Reginald, University of Leicester, 2002.

'Have you forgotten yet?': shellshock, trauma and the memory of the Great War in Britain, 1914-1930, Reid, Fiona, University of the West of England, Bristol, 2005.

'The Report on her Transfer was Shell Shock'. A Study of the Psychological Disorders of Nurses and Female Voluntary Aid Detachments who served alongside the British and Allied Expeditionary Forces during the First World War, 1914-1918, Poynter, Denise J., University of Northampton, 2008.

The trench diseases: the British medical response in the Great War, Atenstaedt, Robert Leslie, University of Oxford, 2005.

Shell-shock in First World War Britain: an intellectual and medical history, c.1860-c.1920, Loughran, Tracey Louise, Queen Mary College, 2006.

Sickness and service: the British Army and the First World War, Hill, Christine Ann, University of Central Lancashire, 2004.

Mobilizing charity: non-uniformed voluntary action during the First World War,Grant, Peter Russell, City University, 2012

Histoire culturelle

First World War memorials, commemoration and community in North East England, 1918-1939, Coss, Denise, Durham University, 2012 
The First World War and narratives of heroic and domestic masculinity in Britain, 1915-1937, Meyer, Jessica, University of Cambridge, 2004.

Engendering race: Jamaica, masculinity and the Great War, Smith, Richard William Peter, University of North London, 2000.

In the shadow of war: continuities and discontinuities in the construction of the masculine identities of British soldiers, 1914 - 1924, Millman, Margaret, University of Greenwich, 2002.

Mobilising 'Etappenhelferinnen' for service with the military: gender regimes in First World War Germany, Schönberger, Bianca, University of Oxford, 2002.

The relationship between Ford, Kipling, Conan Doyle, Wells and British propaganda of the First World War, Jain, Anurag, Queen Mary, University of London, 2009.

Unknown soldiers: Donald Hankey and 'A student in arms'. Davies, Ross.: University of Oxford: 2000.

Dark earth, dark heavens: British apocalyptic writing in the First World War and its aftermath. Hilbert, Ernest Andrew, University of Oxford, 2000.

The Great War and the historical imagination: European intellectuals and the meaning of the past, c. 1914-1937, Aldridge, Ross, University of Reading, 2003.

The truth about the war: canon formation and canonicity in Great War poetry, Harrison, Louise, University of Keele, 2002.

Representations of soldiering: British army uniform and the male body during the First World War, Tynan, Jane, University of the Arts London, 2009.

"A bright memory to remain": the life and works of Charles Sims ${ }^{100}$ RA (1873-1928), Holmes, H. Cecilia, Northumbria University, 2005.

Sights of battle, art and war in Britain, c.1885-1919, Shaw, G.,, University of Nottingham, 2007.

Writing disenchantment: the development of first world war prose, 1918-1930, Frayn, Andrew John, University of Manchester, 2008.

A Supreme Fire of Thought and Spirit: Modernist Patterns of Cultural Renewal in First World War Britain, Jackson, Paul, Oxford Brookes University, 2007.

Collaborators and dissidents: aspects of British literary publishing in the First World War, 1914-1919, Gassert, Imogen L., University of Oxford, 2001.

The British Short Story of the First World War: Form, Function, and Canonisation, Einhaus, Ann-Marie, Durham University, 2010.

'Neither beasts nor gods but men': constructions of masculinity and the image of the ordinary British soldier or 'Tommy' in the First World War art of C.R.W. Nevinson (1889-1946); Eric Henri Kennington (1888-1960) and Charles Sargeant Jagger (1885-1934), Black, Jonathan Andrew Alexander, University College London, 2003.

'Jerusalem in ragtime': reconstructions of 'the Jew' in First World War Britain, Pendlebury, Alyson Jane, University of Southampton, 2001 
Craftsman and Client: the official commissions of Edward Carter Preston ${ }^{101}$, Bampton, Maureen Ann, University of Liverpool, 2007.

'England may keep faith': Two Irish women poets and the great war, Winterson, Kieron, University of Liverpool, 2008.

Beyond a "man's house" women's poetic response to the Great War, Banerjee, Argha Kumar, University of Sussex, 2006.

Female Writers of the First World War, Shearer, Joanna Marian, Oxford Brookes University, 2007.

Marianne's Chroniclers: The Political Journalism of Selected French Female Writers of the First World War, Shearer, Joanna Marian, Oxford Brookes University, 2007.

Women's poetry of the First World War: songs of wartime lives, Newman, Vivien B. E., University of Essex, 2004.

Strategic narratives: American women writers and the First World War, Nolan, Elizabeth, Manchester Metropolitan University, 2005.

Representations of the First World War in British popular culture, 1918-1998, Todman, Daniel, Cambridge University, 2003.

The First World War and popular literature, MacCallum-Stewart, Esther, University of Sussex, 2005.

The big show: cinema exhibition and reception in Britain in the Great War, Hammond, Michael, Southampton Solent University, 2001.

The Great War on the small screen: a cultural history of the First World War on British television, 1964-2005, Mahoney, Emma, University of Kent at Canterbury, 2006.

British children's books and the first world war 1914-2007, Budgen, David, University of Kent, 2010.

A war remembered: commemoration, battlefield tourism and British collective memory of the Great War, Edwards, Peter John, University of Sussex, 2004.

Adoptive kinship and the British League of Help: Commemoration of the Great War through the adoption of French communities, Lewis, Bryan F., University of Reading, 2006.

The commemoration of the Great War in Belfast, Ulster and Northern Ireland, 1918-1939, Manson, Christopher John Matthew, University of Ulster, 2005

The memorialisation of the Great War in Folkestone, Canterbury and Dover, 1918-24, Donaldson, Peter, University of Kent, 2005.

Unionists and Great War commemoration in the north of Ireland, 1914-1939: people, places and politics, Switzer, Catherine Louise, University of Ulster, 2005.

'With God for Kaiser and fatherland': The commemoration of the great war in the first Austrian republic, 1918-1934, Edgecombe, Catherine, University of Southampton, 2008.

'Unconquerable manhood': memory, masculinity and the commemoration of the First World War in British visual culture 1914 to 1930, Koureas, Gabriel, Birkbeck, 2004. 
Dying for the Fatherland: the remembrance of the fallen German-Jewish soldiers of the First World War, 1914-1978, Grady, Tim, University of Southampton, 2006.

Footsteps across time: the evolution, use and relevance of battlefield visits to the British Armed Forces, Caddick-Adams, Peter, University of Cranfield, 2010.

God, Grief and Community: Commemoration of the Great War in Huddersfield, c. 1914-1929, Brook, Anne Christine, University of Leeds, 2009.

Medievalism in the commemoration of the Great War in Britain and Germany, 1914-1939, Goebel, Stefan, University of Cambridge, 2001.

Battlefield tourism: meanings and interpretations, Miles, Stephen Thomas, University of Glasgow, 2012

Art of a second order': the First World War from the British home front perspective, Roberts, Richenda M., University of Birmingham, 2013

Memory, entertainment, propaganda: the Great War and German popular cinema, 1933-1945, Alberts, P. P., University of Salford, 2013

\section{NOTES}

1. Site consulté le 11 mars 2014.

2. Max Hastings, Catastrophe, Europe Goes to War 1914, Londres, William Collins, 2013.

3. Chistopher Clark, The Sleepwalkers: How Europe Went to War in 1914, Harmondsworth, Penguin 2013.

4. Storm of Steel, nouvelle édition, Harmondsworth, Penguin, 2004

5. Traduction française, Paris, La Découverte, 1988.

6. Eric Hobsbawm, op. cit., p. 145.

7. Gary Sheffield, Forgotten Victory - the First World War, Myths and Realities, Londres, Review Press, 2001; The Chief: Douglas Haig and the British Army, Londres, Aurum, 2011.

8. The Last Great War, British Society and the First World War, Cambridge, Cambridge University Press, 2008.

9. Arthur Marwick, The Deluge, British Society and the First World War, Londres, Bodley Head, 1965

10. Keith Battarbee, "The Quest for Coherence and Discipline" in La Civilisation: objet, enjeux, méthodes, revue Babel N 9, 2004.

11. John Mullen, 'The Show must go on' La Chanson populaire en Grande-Bretagne pendant la Grande Guerre 1914-1918, Paris, L'Harmattan, 2012.

12. Antoine Prost et Jay Winter, Penser la Grande Guerre, Paris, Editions du Seuil, 2004.

13. op. cit., p. 49.

14. Antoine Prost et Jay Winter, op. cit., p. 16.

15. Lloyd George, David, War Memoirs of David Lloyd George, (7 tomes), Londres, Oldham, 1936.

16. Pierre Purseigle, « Ecrire l'histoire du Déluge. Histoire et expérience britannique de la Grande Guerre » in Histoire@politique. Politique, Culture, Société, N²2, janvier-avril 2014,p 2

17. Londres, Harper Perennial, 2005.

18. Londres, Macmillan, 1980.

19. Ilana Bet-El, Conscripts, Forgotten Men of the Great War, Stroud, Sutton, 1999.

20. Richard Van Emden, Prisoners of the Kaiser, Barnsley, Pen and Sword, 2000.

21. Richard Van Emden, The Soldiers' War: the Great War through Veterans' Eyes, Londres, Bloomsbury, 2008. 
22. William Philpott, Bloody Victory, the Sacrifice on the Somme, Londres, Abacus, 2010.

23. Stewart Cameron, A Very Unimportant Officer, Londres, Hodder \& Stoughton, 2009; E. W. Hermon, et Anne Nason, For Love and Courage, Letters Home from the Western Front, Londres, Preface, 2008; Edward Campion Vaughan, Some Desperate Glory, The Diary of a Young Officer, 1917, Londres, Pen \& Sword, 2010 (1981).

24. Private Frank Richards, Old Soldiers Never Die, Uckfield, Naval and Military Press, s.d

25. Harry Patch, The Last Fighting Tommy, Londres, Bloomsbury,2008 ; Henry Allingham, et Denis Goodwin, Kitchener's Last Volunteer, Edimbourg, Mainstream Publishing, 2008.

26. Bill Lamin, Letters from the Trenches, A Soldier of the Great War, Londres, Michael O'Mara, 2009 ; Duncan Barrett, (dir.), The Reluctant Tommy, Ronald Skirth's Extraordinary Memoir of the First World War, Londres, Macmillan, 2010 ; Richard Van Emden, Sapper Martin, The Secret Great War Diary of Jack Martin, Londres, Bloomsbury, 2010.

27. Par exemple il a accueilli en 2011 un colloque organisé par le « Mouvement pour l'abolition de la guerre » sur le thème de « l'histoire de la paix ».

28. Max Arthur, Forgotten Voices of the Great War, Londres, Ebury, 2002; Joshua Levine, Forgotten Voices of the Somme, Londres, Ebury, 2008. Lyn Smith, Forgotten voices of the Holocaust, Londres, Ebury, 2005; Joshua Levine, Forgotten voices of Dunkirk, Londres, Ebury, 2010; Joshua Levine, Forgotten voices of the Blitz and the battle for Britain, Londres, Ebury, 2006; Hugh MacManners, Forgotten voices of the Falklands, Londres, Ebury, 2007.

29. La série comprend, entre autres ouvrages: Stephen Snelling, VCs of the First World War : Gallipoli, Stroud , History, 2010 ; Gerald Gliddon VCs of the First World War : 1914, Stroud, Sutton, 1994 ; Stephen Snelling VCs of the First World War: The Naval VCs, Stroud, Sutton, 2002 ; Gerald Gliddon VCs of the First World War : Arras \& Messines, 1917 Stroud, Sutton, 1998 ; Gerald Gliddon, VCs of the First World War : Spring Offensive 1918, Stroud, Sutton, 1997.

30. Mais pas toujours. L'ouvrage de Margaret Macmillan: The War that Ended Peace: How Europe Abandoned Peace for the First World War, Londres, Profile Books, 2013 défend l'importance de causes politiques, culturelles et technologiques.

31. Christopher Clark, The Sleepwalkers, How Europe Went to War in 1914, Londres, Allen Lane, 2012.

32. Antoine Prost et Jay Winter, op. cit., p. 30.

33. Lyn Macdonald, The Roses of No Man's Land, Londres, Penguin, 1993 (1980); Alison S. Fell et Christine E. Hallett (dirs) First World War nursing : new perspectives, New York, Routledge, 2013.

34. Will Ellsworth-Jones, We Will Not Fight...: The Untold Story of World War One's Conscientious Objectors, Londres, Aurum Press 2008.

35. Michael Moynihan, God on Our Side -the British Padre in World War One, Londres, Secker and Warburg, 1983; Michael Snape et Edward Madigan (dirs), The clergy in khaki: new perspectives on British Army chaplaincy in the First World War, Farnham, Ashgate, 2013.

36. Gail Braybon et Penny Summerfield, Out of the Cage, Women's Experience in Two World Wars, Londres, Pandora Press, 1987 ; Susan R. Grayzel, Women and the First World War, Harlow , Longman, 2002 ; Margaret Higonnet et al., (dirs) Behind the Lines: Gender and the Two World Wars, New Haven,Yale University Press, 1987; Gail Braybon, Women Workers in the First World War, Londres, Croom Helm, 1981; Kate Adie, Fighting on the Home Front: The Legacy of Women in World War One, Londres, Hodder and Stoughton, 2013

37. Thomas Cummins Kennedy, The Hound of Conscience: a History of the No-conscription Fellowship, 1914-1919, Fayetteville, University of Arkansas Press,1981; Nigel Keohane, The party of patriotism: the Conservative Party and the First World War, Farnham, Ashgate, 2010.

38. John Horne, Labour at War, France and Britain, 1914-1918, Oxford, Clarendon Press, 1991

39. Anthony Burton, The Workers' War: British Industry and the First World War, Londres, The History press, 2014 and Jeremy Higgins, Great War Railwaymen : British Railway Company Workers at War 1914-1918, Londres, Uniform Press, 2014.

Revue Française de Civilisation Britannique, XX-1 | 2015 
40. Mais sur des periodes un peu plus longues, il y a Gerry Rubin, War, law and labour : the Munitions Acts, state regulation and the unions, 1915-1921, Oxford, Clarendon Press, 1987 et Iain McLean, The Legend of Red Clydeside, Edimbourg, Donald, 1983.

41. Arthur Marwick, The Deluge: British Society and the First World War Londres, Macmillan, 2006 (1965).

42. A. J. P. Taylor, The First World War - an Illustrated History, Harmondsworth, Penguin, 1966.

43. J. M. Bourne, Britain and the Great War, Londres, Hodder \& Stoughton, 1989.

44. Richard Van Emden, et Steve Humphries, All Quiet on the Home Front, an Oral History of Life in Britain during the First World War, Londres, Headline, 2003.

45. http://www.livesofthefirstworldwar.org

46. Antoine Prost et Jay Winter, op. cit., p. 42

47. Pour l'histoire de l'histoire culturelle, voir l'ambitieux livre de Philippe Porrier: Les enjeux de l'histoire culturelle, Paris, Editions du Seuil, 2004.

48. Pour une rare défense récente des "grandes narratives", voir Neil Faulkner, "La Somme, une bataille pour l'empire et le profit » in Henry Daniels et Nathalie Collé-Bak, (dirs), 1916, La Grande-Bretagne en guerre, Nancy, Presses Universitaires de Nancy, 2007.

49. Le directeur de la série est Jay Winter et elle comprend des titres tels que Violence against Prisoners of War in the Frirst World War de Heather Jones, Capital Cities at War, dirigé par Jay Winter, China and the Great War de Guoqi Xuand The Great War and Medieval Memory: War, remembrance and medievalism in Britain and Germany 1914-1940 and The Great War and Urban Life in Germany de Roger Chickering.

50. Cette collection comprend des titres tels que Civvies : Middle-class men on the English Home Front, de Laura Ugolini, Healing the Nation :Soldiers and the Culture of Caregiving in Britain during the Great War, The Secret Battle:Emotional Survival in the Great War, et The Stomach for Fighting: Food and the Soldiers of the Great War, de Rachel Duffett.

51. Une défense de l'histoire contre ces mises en cause peut être trouvée dans l'ouvrage de Richard Evans, In Defence of History, Londres, Granta, 2000, voir mon compte rendu dans la revue Quaderna : http://quaderna.org/richard-j-evans-in-defence-of-history-londres-granta-2000/

52. Keith Jenkins, On 'What is history ?' From Carr and Elton to Rorty and White, Abingdon, Routledge,1995.

53. Auteur notamment de What is History, traduit en français E. H. Carr, Qu'est-ce que l'histoire? (1961), Paris, 10-18, 1996

54. Auteur notamment de G. R. Elton, The Practice of History (1967), Londres, Wiley-Blackwell, 2001.

55. P 10

56. $P 9$

57. Ilana Bet-El, op.cit., p. 61.

58. Rachel Duffett, The Stomach for Fighting : Food and the Soldiers of the Great War, Manchester, Manchester University Press, 2012.

59. Paul Fussell, The Great War and Modern Memory, Oxford, Oxford University Press, 2000 (Première parution 1975).

60. C. Delacroix, F. Dosse, P. Garcia et N. Offenstadt, Historiographies 1: Concepts et débats, Paris, Gallimard, 2010, pp 220-231.

61. Les exemples sont très nombreux, mais on peut citer : Michael Keren and Holger H. Herwig War Memory and Popular Culture: Essays on Modes of Remembrance and Commemoration, Jefferson, Macfarland, 2009 ; Catherine Switzer, Unionists and Great War Commemoration in the North of Ireland, 1914-1939, Dublin, Irish Academic Press, 2007

62. Charles-Robert Ageron et Pierre Nora, Les Lieux de mémoire, Paris, Gallimard, 1997. 
63. Mais on trouve des antécédents à leur travail chez de tels historiens que John Terraine dans les années 1960, auteur notamment de Douglas Haig The Educated Soldier, Londres, Hutchinson, 1963.

64. Alan Clark, The Donkeys, Londres, Pimlico, 1991 (1961).

65. Gordon Corrigan, Mud Blood and Poppycock, Londres, Cassell, 2003 ; Sepoys in the Trenches: The Indian Corps on the Western Front, 1914-15, Londres, Spellmount, 2006; Loos 1915: The Unwanted Battle, Londres, Spellmount, 2005.

66. Niall Ferguson, The Pity of War, Londres, Allen Lane, 1998; Empire, How Britain Made the Modern World, Londres, Allen Lane, 2003.

67. Gary Sheffield, Forgotten Victory - the First World War, Myths and Realities, Londres, Review Press, 2001; The Chief: Douglas Haig and the British Army, Londres, Aurum, 2011.

68. http://www.bbc.co.uk/history/worldwars/wwone/

69. Sur un échantillon représentatif de près de 2000 britanniques. https://yougov.co.uk/ news/2014/01/09/wwi-generals-let-down-troops/ (Consulté le 5 janvier 2015)

70. Sunday Times 7 novembre 2004. Ces sentiments l'ont mené à refuser les funérailles d'état qui lui étaient proposées. Lors de ses funérailles, le cercueil était porté par des soldats français, belges et allemands, accompagné d'une chanson antimilitariste des années 1950.

71. http://ethos.bl.uk/Home.do

72. Le total ne fait pas $100 \%$ car j'ai arrondi au dixième près.

73. La présence de quelques thèses qui ne rentrent pas dans les sous-catégories impose que le total des sous-catégories n'est pas exactement le même que celui des catégories.

74. Contrairement à la plainte qu'on lit fréquemment dans les forums en ligne d'aficionados des études de la Première Guerre, selon laquelle «les intellectuels» auraient définitivement abandonné l'histoire militaire.

75. «What alternative punishment is there?» : military executions during World War I, Oram, Gerard Christopher, The Open University, 2000.

76. Par exemple: British Catholic identity during the First World War : the challenge of universality and particularity, Finlay, Katherine, University of Oxford, 2004 ou The First World War and Voluntary Recruitment: A Forum for Regional Identity? An analysis of the nature, expression and significance of regional identity in Hull 1900-1916, Townsley, Helen, University of Sussex, 2007.

77. Par exemple: 'Pitied but distrusted' : discourses surrounding British widows of the First World War, Smith, Angela, University of Sunderland, 2007.

78. Within their sphere? Women's correspondence to Aberdeen daily newspapers, 1900-1918, Pedersen, Sarah, Robert Gordon University, 2004.

79. 'The Report on her Transfer was Shell Shock'. A Study of the Psychological Disorders of Nurses and Female Voluntary Aid Detachments who served alongside the British and Allied Expeditionary Forces during the First World War, 1914-1918, Poynter, Denise J., University of Northampton, 2008

80. 'Have you forgotten yet?' : shellshock, trauma and the memory of the Great War in Britain, 1914-1930, Reid, Fiona, University of the West of England, Bristol, 2005.

81. Problems, politics and personalities in the treatment of mental and nervous casualties in the British Army 1914-1918. Incorporating a statistical and analytical study of 500 case histories, Hopkins, John Reginald, University of Leicester, 2002.

82. Collaborators and dissidents : aspects of British literary publishing in the First World War, 1914-1919, Gassert, Imogen L., University of Oxford , 2001.

83. The British Short Story of the First World War: Form, Function, and Canonisation, Einhaus, Ann-Marie, Durham University, 2010.

84. Craftsman and Client: the official commissions of Edward Carter Preston, Bampton, Maureen Ann, University of Liverpool, 2007. 
85. Representations of soldiering: British army uniform and the male body during the First World War, Tynan, Jane ,University of the Arts London, 2009.

86. The Great War on the small screen : a cultural history of the First World War on British television, 1964-2005, Mahoney, Emma, University of Kent at Canterbury, 2006.

87. The big show : cinema exhibition and reception in Britain in the Great War, Hammond, Michael, Southampton Solent University, 2001.

88. British children's books and the first world war 1914-2007, Budgen, David, University of Kent, 2010.

89. Peter Bailey, Leisure and class in Victorian England: Rational Recreation and the Contest for Control 1830 to 1885 , Londres, Routledge, 1978.

90. Par exemple: A war remembered : commemoration, battlefield tourism and British collective memory of the Great War, Edwards, Peter John, University of Sussex, 2004

91. The memorialisation of the Great War in Folkestone, Canterbury and Dover, 1918-24, Donaldson, Peter, University of Kent, 2005.

92. Medievalism in the commemoration of the Great War in Britain and Germany, 1914-1939, Goebel, Stefan, University of Cambridge, 2001.

93. http://www.history.ac.uk/makinghistory/resources/interviews/Hobsbawm_Eric.html

94. http://www.theses.fr/

95. Pierre Purseigle, « Ecrire l'histoire du Déluge. Histoire et expérience britannique de la Grande Guerre » in Histoire@politique. Politique, Culture, Société, Nº 22, janvier-avril 2014.

96. Pierre Purseigle, op. cit., p. 9.

97. Il y a évidemment de notables exceptions, telles que Arthur Marwick, Total War and Historical Change : Europe 1914-55, Buckingham, Open University Press, 2001.

98. Voir Richard J Evans In Defence of History, Londres, Granta, 2000, p.244.

99. Richard Ned Lebow, Archduke Franz Ferdinand Lives!: A World without World War I, Londres, Palgrave Macmillan, 2014

100. Un artiste de guerre, sui a perdu son fils aîné dans les combats.

101. Sculpteur et concepteur de médailles

\section{RÉSUMÉS}

Quelles sources les historiens britanniques de la Première Guerre ont-ils utilisées? Quelles questions ont-ils posées? Cet article retrace en détail les trois configurations historiographiques identifiées par Prost et Winter et explore des controverses historiographiques récentes, avant de procéder à une analyse thématique détaillée des 103 thèses sur la Grande Guerre soutenues au Royaume-Uni depuis 2000.

Few periods of history have been as much studied as has the First World War. This article traces the historiography of the Great War in Britain, concentrating on the last 50 years. The period of domination of military and diplomatic history gave way in the sixties to social history and 20 years later to cultural history, but what precise effect did this have on the questions asked and the sources used by historians? After bringing us up to date with recent historioraphical debates, the article goes on on to analyse the themes dealt with in the 103 doctoral theses on the war produced in Britain since the year 2000. 
INDEX

Mots-clés : Première Guerre mondiale, historiographie, Royaume-Uni, thèses doctorales

Keywords : First World War, historiography, British history, doctoral theses, United Kingdom

\section{AUTEUR}

JOHN MULLEN

Université Paris-Est Créteil 